\title{
Slip ring test assembly with increased breakdown voltage limit for high-voltage bus satellites
}

F. Avino, ${ }^{1, a)}$ B. Gaffinet, ${ }^{1}$ D. Bommottet, ${ }^{2}$ A. Howling, ${ }^{1}$ and I. Furno ${ }^{1}$

1) Ecole Polytechnique Fédérale de Lausanne (EPFL), Swiss Plasma Center (SPC), CH-1015 Lausanne, Switzerland

2) RUAG Space, CH-1260 Nyon, Switzerland.

(Dated: 5 May 2020)

In this work, a setup including a passive gas breakdown mitigation technique for satellite slip ring assemblies (SRAs) is introduced and tested. The setup features a central conducting ring, separated from two external conducting limiting discs (LDs) by insulating rings. The characteristic breakdown curves are measured as a function of the surrounding pressure by biasing the central ring and setting the LDs at a variable voltage. The minimum breakdown voltage is increased from $\sim 420 \mathrm{~V}$ to $\sim 860 \mathrm{~V}$ by biasing the LDs at one half of the voltage applied to the central ring. This solution is further applied on a test-SRA that includes the main features of a standard cylindrical SRA: a stack of conducting rings on which conducting brushes slip to ensure the electrical contact between moving components. The minimum of the breakdown curve is raised from $\sim 400 \mathrm{~V}$ for the grounded limiting discs to $\sim 600 \mathrm{~V}$ for the passively-biased limiting discs. This result confirms the possibility to improve SRA protection for the entire pressure range encountered by a satellite during its operational life, from atmospheric pressure to in-orbit high-vacuum, meeting the requirements for the next generation of satellites.

a)fabio.avino@cern.ch 


\section{INTRODUCTION}

The development of high-voltage (HV) bus systems for satellites in the $300-600 \mathrm{~V}$ range is currently ongoing to meet the requirements of new generation high-power thrusters. ${ }^{1}$ The risk of electrical breakdown ${ }^{2-5}$ for solar panels ${ }^{6}$ and for slip ring assemblies (SRAs) is a crucial aspect of the feasibility assessment. The SRA is the component of the power transmission line that allows the power transfer between the rotating solar panels and the Power Control Unit of the satellite. The complexity of a standard SRA design with a stack of gold-plated brushes slipping on gold-plated rings makes gas breakdown mitigation a challenging problem. The voltage differences between conducting rings, brushes, and the housing may lead to electrical breakdown and possible failure. Therefore, electrical breakdown must be avoided from atmospheric pressure in the case where HV is switched-on during launch, to the high-vacuum in the operational orbit. This is due to the pressure uncertainty during the de-pressurization phase of the satellite and to the local fluctuations

of pressure that could occur during the satellite life because of degassing, the impact of micro-meteoroids or the thruster gas back-flow.

First studies to more effectively inhibit breakdowns in SRA were undertaken in previous works, ${ }^{7}$ clarifying with a physics-oriented approach the role of the direct-current (DC) gas breakdown mechanisms underlying the breakdown curve of a standard SRA. ${ }^{8}$ The safe zone of operation of a SRA was improved in terms of pressure by implementing grounded limiting discs (LDs) with extended diameter, which act as partial Faraday screens. This design was proven to be effective on the low pressure branch of the breakdown curve, without affecting its voltage minimum. To meet the target voltage of $600 \mathrm{~V}$ under investigation by the space community, an improved configuration is herein presented. The potential of the LDs is optimized at half the value of the HV ring by using a voltage divider of two equal resistances. The relative bias between the HV-ring and the LDs is shown to be the key parameter that determines the breakdown at high pressures. This allows to raise the minimum of the breakdown curve from $\sim 400 \mathrm{~V}$ up to $\sim 600 \mathrm{~V}$ in a newly developed test-SRA.

In Sec. II, we introduce the experimental set-up, including the passively-biased LDs solution with the basic assembly and the test-SRA. In Sec. III and IV, the experimental measurements of breakdown curves with the mitigation technique are shown, respectively, for the basic assembly and for the test-SRA. The conclusions are discussed in Sec. V. 


\section{PASSIVELY-BIASED LIMITING DISCS: BASIC ASSEMBLY AND TEST-SRA}

To increase the minimum breakdown voltage of a cylindrical SRA at high pressures, the circuit depicted in Fig. 1 is connected to the setup shown inside the dashed contour, which has been previously discussed in [8] with grounded LDs. This basic assembly is

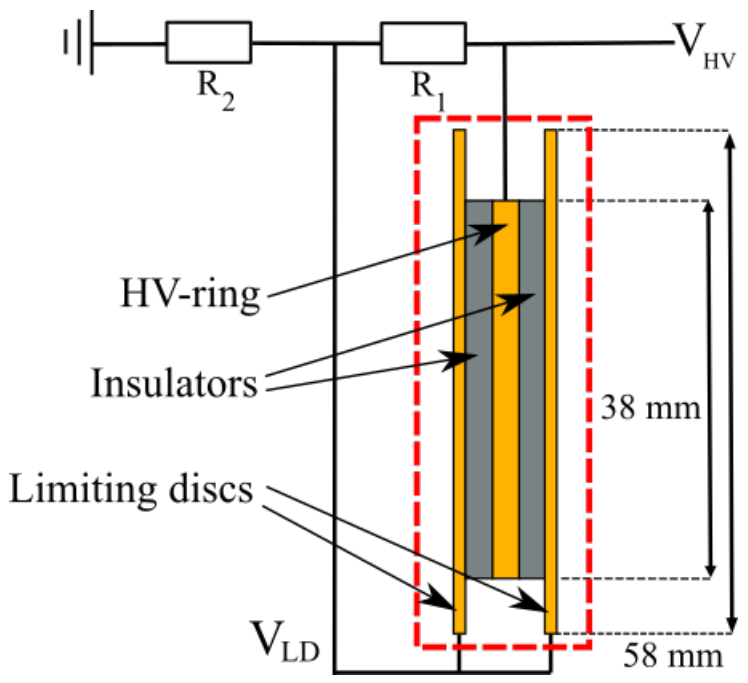

FIG. 1. Schematic design of the basic assembly with the passively-biased LDs configuration.

composed of a central HV-ring of $38 \mathrm{~mm}$ diameter separated by two insulating rings from two conducting LDs of $58 \mathrm{~mm}$ diameter, as indicated in Fig. 1. The bias of the two LDs $\left(V_{\mathrm{LD}}\right)$ is proportional to the central ring voltage $\left(V_{\mathrm{HV}}\right)$ according to the voltage divider composed of two resistances $R_{1}$ and $R_{2}$. Since the physical parameter that determines the breakdown on short paths between the HV-ring and the LDs is their relative voltage ( $V_{\mathrm{HV}}$ - $V_{\mathrm{LD}}$ ), the measured breakdown curves are identified with the parameter $\eta$ such that:

$$
\eta=\frac{R_{2}}{R_{1}+R_{2}}=\frac{V_{L D}}{V_{H V}} \quad \Rightarrow \quad V_{L D}=\eta V_{H V} .
$$

The values of the resistances must be chosen to find a tradeoff between two effects. The ratio $R_{1} / R_{2}$ must be high enough to provide a sufficient voltage difference between $V_{\mathrm{HV}}$ and $V_{\mathrm{LD}}$ to maintain the Faraday screen effect, hampering the HV-ring breakdown at low pressures on long paths towards the grounded enclosure. A value of $R_{1} / R_{2} \gg 1(\eta \simeq 0)$ tends to the grounded LDs solution. At the same time, $R_{1} / R_{2}$ must result in a shift to higher values of both $V_{\mathrm{HV}}$ and $V_{\mathrm{LD}}$ with respect to the surrounding grounded enclosure 
before the breakdown of the $\mathrm{HV}$ ring occurs. $R_{1} / R_{2} \ll 1(\eta \simeq 1)$ leads to $V_{\mathrm{LD}} \simeq V_{\mathrm{HV}}$, therefore losing the Faraday screen effect and also making the breakdown between the LDs and the grounded enclosure the dominant process. The resistances $R_{1}$ and $R_{2}$ must be low enough to allow currents larger than the background Townsend currents to avoid affecting the LDs potential, but high enough to keep these currents below the current limit threshold of breakdown detection. This leads to the selection of resistances of few tens of $\mathrm{M} \Omega$ as discussed in Sec. III, where measurements of breakdown curves with the passively-biased configuration are presented.

To reproduce the complex structure of a satellite SRA, the test-SRA shown in Fig. 2 is developed. It is composed of a conducting housing and a central conducting cylindrical axis on which 36 gold-plated rings of $2 \mathrm{~mm}$ thickness are inserted. Similarly to the basic assembly, these rings are designed to be used in groups of three, arranged along the stack with a central HV-ring of $32 \mathrm{~mm}$ enclosed between two LDs whose diameter progressively increases from $34 \mathrm{~mm}$ to $56 \mathrm{~mm}$. This allows to test the grounded or passively biased configurations for different size of LDs. Consecutive conducting rings are separated by $1 \mathrm{~mm}$ thick insulating rings. Gold-plated brushes mounted on the insulating board on top of the housing slip on the corresponding rings, providing electrical continuity. The brushes are covered with a film of insulator (Kapton), with the exception of the tip in contact with the ring. This is necessary to avoid the breakdown with the rest of the assembly, since the brushes extend beyond the volume screened by the LDs. The printed-circuit board in Fig. 2 (c) allows to independently control the potential of each ring. Breakdown measurements of the test-SRA are presented in Sec. IV.

\section{BASIC ASSEMBLY BREAKDOWN MITIGATION}

Several values of $R_{1} / R_{2}$ are used in the basic assembly in a vacuum chamber to study the effect of biased LDs. A Danbridge $30 \mathrm{kV}$ non-destructive insulation tester type JP30 A is used to reach the breakdown condition. Breakdown voltages for the HV ring are obtained for different pressures, which are measured with two baratrons (2 mbar and 1000 mbar range), a Pirani gauge and a cold cathode gauge. Over-voltage effects are avoided by increasing the voltage at $\sim 10 \mathrm{~V} / \mathrm{s}$. The breakdown curves shown in Fig. 3 (a) are measured for $\eta=[0,0.21,0.26,0.33,0.5]$, corresponding to the resistances indicated in Table I. 




FIG. 2. (a) Side view of the test-SRA including the stack of alternating insulating and conducting rings of progressively increasing diameter, with the gold-plated brushes and one group of three gold-plated rings indicated. (b) Close-up view of the ring stack and the associated brushes. (c) Top view of the assembly showing the printed-circuit board including the soldered connections to the brushes.

\begin{tabular}{|c|c|c|}
\hline$\eta$ & $R_{1}[\mathrm{M} \Omega]$ & $R_{2}[\mathrm{M} \Omega]$ \\
\hline \hline 0.21 & 68 & 18 \\
\hline 0.26 & 68 & 24 \\
\hline 0.33 & 68 & 33 \\
\hline 0.5 & 68 & 68 \\
\hline
\end{tabular}

TABLE I. Implemented resistances for the corresponding values of $\eta$.

We can observe that by increasing $\eta$, the breakdown voltages on the low-pressure region ( $\mathrm{p} \leq 7 \times 10^{-1} \mathrm{mbar}$ ) progressively decrease, indicating a partial loss of the screening effect of the LDs. Contrarily, the high-pressure branch ( $\left.\mathrm{p} \geq 7 \times 10^{-1} \mathrm{mbar}\right)$ features a significant increase for $\eta=0.5$, from $\sim 420 \mathrm{~V}$ to $\sim 860 \mathrm{~V}$. Two separate minima can also be observed at $\sim 3 \times 10^{-1}$ and $\sim 5$ mbar, corresponding to the two breakdown curves on long and short paths, respectively.

We note that breakdowns for different values of $\eta$ occur for the same voltage difference 

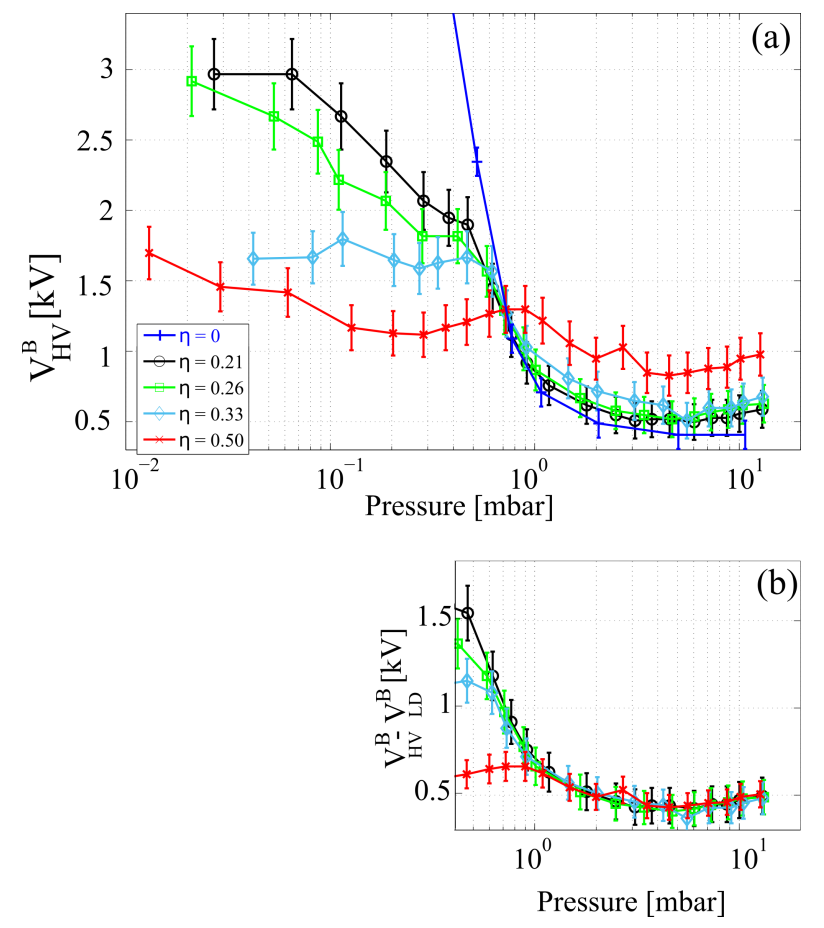

FIG. 3. (a) Breakdown voltage curves of the HV ring for the basic assembly. (b) Difference of voltage between the HV ring and the LDs at the breakdown.

between the $\mathrm{HV}$-ring and the LDs $\left(V_{\mathrm{HV}}-V_{\mathrm{LD}}\right)$ in the high pressure branch, as shown in Fig. 3 (b). This is consistent with the presence of short path breakdowns.

\section{TEST-SRA BREAKDOWN MITIGATION}

The two breakdown curves shown in Fig. 4 are measured in an automated test bench at RUAG Space Nyon. The thermo-vacuum chamber has a base pressure of $\sim 10^{-7}$ mbar. A negative polarity dielectric tester applies a DC voltage to the component under test via two high-voltage feedthroughs. The tester is controlled via a LabVIEW software and interface to automatically measure a complete breakdown curve of the component under study. The adjustable parameters are the minimum and maximum air pressure, the number of pressure points per pressure decade, and the number of measurements for each pressure value. The voltage ramp applied by the dielectric tester features steps of $1 \mathrm{~s}$ duration with the voltage increment determined by the maximum required voltage and the total rampup time. The experiments herein are performed with a voltage ramp up to $1.5 \mathrm{kV}$ of $30 \mathrm{~s}$ total duration, resulting in steps of $50 \mathrm{~V}$. The ramp stops if the $1 \mathrm{~mA}$ threshold current for 
breakdown detection is measured, or when the maximum voltage of $1.5 \mathrm{kV}$ is reached, which is maintained for $4 \mathrm{~s}$ and then reduced back to $0 \mathrm{~V}$ in $2 \mathrm{~s}$. Five values of breakdown voltage are measured for each pressure, and the corresponding standard deviation is shown as error bar. The pressure range from $10^{-1}$ mbar to 7 mbar is sufficient to investigate the minimum of the breakdown curve which is close to 2 mbar.

The breakdown curve for the group of $54 \mathrm{~mm}$ LDs at the ground $(\eta=0)$ has a minimum of the breakdown voltage at $\sim 400 \mathrm{~V}$ close to 2 mbar. By biasing the LDs with $\eta=0.5$,

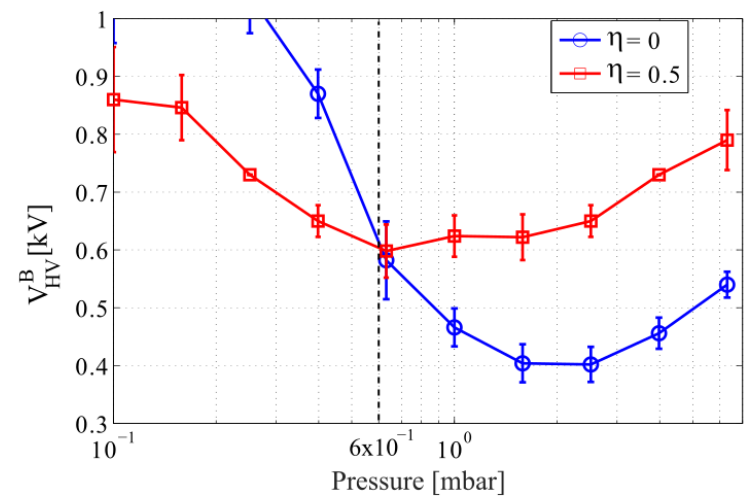

FIG. 4. Breakdown curves of the HV ring for the test-SRA with $54 \mathrm{~mm}$ LDs: in blues circles, the case of grounded LDs $(\eta=0)$, in red squares the curve measured with the passively-biased LDs $(\eta=0.5)$.

lower values of breakdown voltage are observed with respect to that with $\eta=0$ for pressures below $6 \times 10^{-1}$ mbar. This is consistent with the less effective screening of breakdown between the HV-ring and the housing. At $6 \times 10^{-1}$ mbar, the minimum breakdown voltage at $600 \mathrm{~V}$ is reached, which is $50 \%$ higher than that obtained with grounded LDs. For pressure values above $6 \times 10^{-1} \mathrm{mbar}$, the breakdown voltage progressively increases. We note that the breakdown curve for $\eta=0.5$ of the test-SRA does not show two separate minima like that of the basic assembly in Fig. 3 (a). This can be related to the main difference between the two designs in the accessible breakdown paths when the LDs are biased. In the basic assembly, the presence of only one central HV-ring and two LDs limits the available breakdown path to two characteristic lengths on $\sim 10 \mathrm{~cm}$ scale towards the grounded enclosure, and $\sim 1 \mathrm{~cm}$ scale between the HV-ring and the LDs. In the test-SRA, all the path lengths are available given the presence of the grounded stack of conducting LDs and the housing. In particular, the accessibility of short path breakdown between the 
LDs and adjacent grounded components justifies the optimal situation for $\eta=0.5$ when the conditions for the breakdown ignition are satisfied at the same time between the HV-ring and the LDs, and between the LDs and the closest grounded components. However, the measured improvement in breakdown voltage between the $\eta=0$ and the $\eta=0.5$ curve of the test-SRA is smaller than what is observed in Sec. III for the basic assembly. Further

studies will focus on this feature. The two resistances in series of $68 \mathrm{M} \Omega$ lead to a current of $\sim 7 \mu \mathrm{A}$ for $600 \mathrm{~V}$ DC. This current is high enough to avoid that the background Townsend currents affect the LDs potential. This corresponds to a power loss of $\sim 4 \mathrm{~mW}$, which would be acceptable in a real satellite.

\section{CONCLUSIONS AND OUTLOOK}

Previous investigations on gas breakdown mitigation in satellite SRAs have led to an improvement of the safe zone of operation with respect to the pressure, but without increasing the minimum breakdown voltage. ${ }^{7,8}$ In this work, a new passive gas breakdown mitigation solution for satellite SRAs is presented to meet the $600 \mathrm{~V}$ of safe operation that is required for the new generation of high-power satellite thrusters. A series of resistances is used to passively bias two conducting LDs that enclose a central high-voltage ring. In this way, the voltage of the LDs rises together with that of the central ring, which allows to raise the minimum voltage of the characteristic breakdown curve of the slip-ring assembly. This approach has been tested both on a basic assembly with three discs featuring the basic elements of a SRA, as well as on a test-SRA composed of all the main components of a real cylindrical slip ring, including gold-plated discs and brushes. The potential of the LDs is optimized at half the value of the HV ring. For the first assembly, the minimum of the breakdown curve is increased from $\sim 420 \mathrm{~V}$ to $\sim 860 \mathrm{~V}$. For the test-SRA, the minimum breakdown voltage is increased from $\sim 400 \mathrm{~V}$ to $\sim 600 \mathrm{~V}$. Based on these results, a prototype SRA could be developed for next generation of high-voltage bus satellites. 


\section{ACKNOWLEDGMENTS}

This work is part of the High Voltage - Electrical Power System Architecture (HV-EPSA) project and it has received funding from the SEFRI under grant agreement number 15.0340, in the framework of the European Union's Horizon 2020 research and innovation programme. The views and opinions expressed herein do not necessarily reflect those of the European Commission. The authors wish to acknowledge the support of the SPC and RUAG Space Nyon technical teams.

\section{REFERENCES}

${ }^{1}$ Leporini A., Giannetti V., Andreussi T., Pedrini D., Rossodivita A., Piragino A., Andrenucci M., and Estublier D., Development of a $20 \mathrm{~kW}$-class Hall effect thruster, Proceedings of Space Propulsion, (2016).

${ }^{2}$ Osmokrovic P., Vujisic M., Stankovic K., Vasic A., and Loncar B., Plasma Sources Sci. Technol. 16, 643 (2007).

${ }^{3}$ Maglaras L.A., and Maglaras A.L., Transactions on Power Systems 3, 103 (2008).

${ }^{4}$ Petrović Z.Lj., Skoro N., Marié D., Mahony C.M.O., Maguire P.D., Radmilović M., and Malović G., J. Phys. D: Appl. Phys. 41, 194002 (2008).

${ }^{5}$ Lisovskiy V.A., Koval V.A., and Yegorenkov V.D., Phys. Lett. A 375, 1986 (2011).

${ }^{6}$ Bonin G., Orr N., Zee R.E., and Cain J., Solar Array Arcing Mitigation for Polar Low-Earth Orbit Spacecraft, 24th Annual AIAA/USU Conference on Small Satellites (2010).

${ }^{7}$ Schnyder R., Howling A.A., Bommottet D., and Hollenstein C., J. Phys. D: Appl. Phys. 46, 285205 (2013).

${ }^{8}$ Avino F., Martens P., Bommotet D., Howling A.A., Furno I., Aerospace Sci. and Technol. 85, 229 (2019). 